\title{
Research on Further-Supporting Mechanism of Academic Science and Technology Competition Achievements for Science and Engineering Majors
}

\author{
Jinhong Li ${ }^{1}$, Kangpei Zhao ${ }^{2 *}$ \\ ${ }^{1}$ School of Mathematics and Statistic, Qilu University of Technology (Shandong Academy of Sciences), Jinan, China \\ ${ }^{2}$ School of Construction Machinery, Shandong Jiaotong University, Jinan, China \\ Email: lijinhong@qlu.edu.cn, *kpzhao@sdjtu.edu.cn
}

How to cite this paper: Li, J. H., \& Zhao, K. P. (2021). Research on Further-Supporting Mechanism of Academic Science and Technology Competition Achievements for Science and Engineering Majors. Advances in Applied Sociology, 11, 482-491.

https://doi.org/10.4236/aasoci.2021.1110042

Received: September 14, 2021

Accepted: October 24, 2021

Published: October 27, 2021

Copyright $\odot 2021$ by author(s) and Scientific Research Publishing Inc. This work is licensed under the Creative Commons Attribution International License (CC BY 4.0).

http://creativecommons.org/licenses/by/4.0/

\begin{abstract}
Based on the relevant national policies on Innovation and Entrepreneurship (hereinafter referred to as I\&E) education, the formulation of further-supporting policies for excellent competition achievements in Qilu University of Technology is studied in this paper. Additionally, the exhibition platform of competition achievements is also explored to carry out the quality tracking and evaluation system for further project-supporting. Theoretically, the exploration and research on further-supporting can provide guidance for improving the quality and efficiency of I\&E of Qilu University of Technology.
\end{abstract}

\section{Keywords}

I\&E, Achievement Further-Supporting, Mechanism

\section{Introduction}

The Opinions of the Ministry of Education on Vigorously Promoting I\&E Education in Colleges and Universities and College Students' Independent Entrepreneurship clearly points out that college students are one of the groups with the most I\&E potential. Carrying out I\&E education in colleges and universities for students to start their own businesses is a major strategic measure for the education system to deeply study and practice the scientific outlook on development and serve the construction of an innovative country. It is an important way to deepen the teaching reform of higher education and cultivate students' innovative spirit and practical ability. What's more, it is a significant measure to promote 
employment by entrepreneurship and encourage the full employment of college graduates. The I\&E practice based on various college students' academic science and technology competition platforms have become an effective approach to stimulate college students' innovation enthusiasm and improve their innovation ability, which has a far-reaching impact on talent training and construction. Therefore, it is imperative to stress the cultivation of students' capacity in practice and entrepreneurship ( $\mathrm{Li}$ et al., 2020; Gu, 2020). The improvement of innovative practice education and the construction of a scientific and effective innovative practice system are not only an effective way to cultivate their innovative spirit and ability, but also the inevitable requirement of reforming the talent training mode.

\section{Analysis on the Current Situation of College Students' Scientific and Technological Innovation Activities}

The high-quality development of higher education puts forward new requirements for talent training, which pays more attention to the cultivation of students' innovation and entrepreneurship ability. Therefore, it is necessary to analyze the current situation of scientific and technological innovation activities and think about how to implement innovation and entrepreneurship education activities.

The current situation of college students' scientific and technological innovation activities is as follows. Firstly, it presents a positive development on the whole. The nation respects scientific and technological talents. In addition, colleges and universities have initially formed a good academic and technological atmosphere for students who have high enthusiasm to participate in various academic and technological innovation activities. Graduates with strong scientific and technological innovation spirit and ability are deeply welcomed by employers. Secondly, the multiple scientific and technological innovation activities have become an academic competition with authority, demonstration and guidance from top to bottom, covering all levels of "Nation-Province-City-School". Thirdly, it is obvious that students who participate in these activities find their participation rewarding. Under the guidance of policy and public opinion, the colleges and universities not only participate in academic scientific and technological innovation activities with wide coverage and large quantity, but also improve their entry quantity and quality in steady pace, which provides a good fundamental environment and driving force for the generation, transformation and utilization of excellent scientific and technological achievements. (Du et al., 2019; Li, 2016).

Discipline competition is not only an effective test for scientific and technological innovation education in colleges and universities, but also an important way to present the scientific and technological innovation. Driven by competition, the construction of scientific and technological innovation education system in colleges and universities is featured by autonomy, progressiveness, coor- 
dination and sustainability (Yan et al., 2021). In the process of promoting discipline competition, the cooperation and exchange with enterprises and research institutions should be strengthened as much as possible, so as to sprout new impetus for innovation and entrepreneurship. Many useful attempts have been made in this regard. Some scholars focus on the universal, critical and forward-looking technology and use the strategic, service, and benefit synergy, to integrate the research of scientific institutes, the incubation of incubators, and industrialization of industry bases to be an organic entirety which produces collaborative innovation (Zhang, 2017; Fang \& Montazeritabarar, 2020). As one of the major approaches, the academic promotion can maintain and enhance the quality and efficiency of higher education. Under this background, there is still room to improve for the domestic college students' innovative activity practice system. Further improvement needs to be performed in the construction of practice system. For example, the policies and mechanisms of innovative practical education activities need to be further improved, and the operation mode of academic science and technology activities fail to achieve diversified development. As for college students' academic science and technology and I\&E education, the connection between innovation theory and practice is not close enough. What's more, it attaches importance to the guidance of entrepreneurial skills and the early and middle guidance of academic scientific and technological activities, but ignores the cultivation of innovative spirit and ideas and the later improvement, transformation and application of achievements. At present, from the full perspective of "early-middle-later" in the academic science and technology activities, the teaching and practice of innovative system is operated in the "early" stage to lay a sound theoretical and methodological foundation for students to participate in academic science and technology competitions. In the "middle" stage, activities such as selecting team members, organizing competition, competition guidance and on-site guidance are carried out for specific competitions. At present, colleges and universities pay more heed to the first two stages and are trying to create conditions for students to participate in the competition, providing good theories, methods, hardware, software, teachers and financial conditions. However, in the "later" stage, that is, after the competition, questions presented are not studied continuously, which could not help to form relatively systematic achievements in academic and scientific and technological activities. Also, the problems exposed in the competition are not well utilized, which means they cannot be modified, improved, transformed and applied. Meanwhile, the schemes and characteristics of the same type of entries from brother colleges fail to integrate with their own works deeply, and the further promotion of the project, the transformation and application can hardly be achieved. Therefore, it is urgently necessary to conduct further-supporting and transformation and application for systematic academic scientific and technological achievements in later stage (Nie, 2019). 


\section{Research Ideas and Implementation Paths of Further-Supporting Project in the Later Stage of College Students' I\&E}

According to the relevant policies on I\&E education practice issued by the nation and Shandong province, based on the current situation of college students' academic science and technology competition in Qilu University of technology, the quality and efficiency of I\&E education for students in relevant majors are expected to be improved by conducting multi-directional research from the perspective of innovative and applied talents training in science and engineering, building excellent achievement further-supporting policies suitable for academic science and technology competition in Science and Engineering majors, constructing a platform for project achievement exhibition and transformation, formulating a quality tracking and evaluation system for further-supporting project. In the process of research, the authors pay attention to learning the experience of I\&E education activities from brother colleges and universities, with comprehensive consideration for actual situation of Qilu University of Technology. On the basis of summarizing the theoretical system and current situation of I\&E education, it has combined many scientific research institutes, enterprises and public institutions and social incubation platforms, including College of Entrepreneurship, School of Mathematics and Statistics, School of Mechanical and Automotive Engineering, School of Electrical Engineering, School of Chemistry and Pharmaceutical Engineering, School of Computer Science and Technology, Shandong Jiaotong University, Shandong Academy of Sciences, Shandong Institute of Artificial Intelligence, Shandong Inspur Group. Relying on the excellent academic science and technology competition achievements of these teams, reasonable project construction objectives are formulated gradually in the implementation of each sub item, which promotes the I\&E quality and the transformation and application of these achievements, and encourages the innovation spirit and ability of students majoring in science and engineering through optimizing the student team and instructor team under the guidance of academic science and technology competition. Including:

\subsection{Formulation of Relevant Policies for Further-Supporting of Academic Competition Achievements}

Referring to the existing policies related to academic science and technology competitions of the university, the characteristics of science, electro-mechanical and information academic competition platforms and project should be considered as a whole to formulate a series of policies for the further-supporting, transformation and application of excellent achievements. Specifically, it includes methods for further-supporting project selection, team construction, fund source and use, process assessment, and incentives for the transformation and application of excellent achievements, etc. It can ensure that the promotion and transformation of achievements from further-supporting project by means of formu- 
lating targeted policies and giving full play to the incentive role of the policy as much as possible.

\subsection{Construction of Achievement Exhibition and Transformation Platform}

It is necessary to build a network platform integrating management, exhibition and industrial "tie-in", in order to prepare in the application and screening of further-supporting project, the dynamic exhibition of achievements, the process assessment and the industrial "tie-in". With the help of website or APP resources, it can not only serve the internal management and assessment of the project, but also be in charge of the connection between research achievements and the industry. The functions that the platform can realize mainly include project application, update of team members and guidance team, exhibition of research progress, submission and review of conclusion materials, exhibition of academic achievements from excellent college students for industry, "tie-in" of achievement industrialization, etc. The level and quality of project achievements are supposed to be improved and transformed through the full establishment of the transformation platform, the process control and the industrial "tie-in" for excellent achievements.

\subsection{Implementation of Further-Supporting and Quality Tracking and Evaluation Systems for the Project}

Attach importance to selection, team optimization, implementation, process control and overall evaluation of the further-supporting project. In the selection part, according to decided methods in advance, the preliminary achievements are confined within present ones in academic science and technology competitions achieved in such categories as sciences, information and technology, mechanical and electrical engineering. The later achievements come to extend to academical and technological ones by other categories of the engineering. The project application is able to carry out both through the expert's recommendation and personal application. In the part of team assembling and refining, with the full consideration of specific requirements for the new engineering and the academic science and technology competitions increasingly featured by disciplinary intersecting and integrating, the works for the competitions are required much higher standard in information, intelligence and program optimization. As a result, the layout of members across the project team needs to be adjusted as well as ensuring that the several aspects above find the corresponded majors; in the meantime, teacher instructors should also embrace the inter-disciplinary knowledge structure, from which it is advised that at least two teachers with different discipline backdrop simultaneously complete the instruction. In the part of implementation, with no much interference, team leader and instructor accountability systems are carried out. In the part of process control, more efforts should be taken in dynamic assessment of the project, and to the transformation platform 
the stage progress report should be submitted for review by the department in charge on its consistency with programming. The expert will get involved into this part, implement knockout of the last system and premium fund goes to the excellent project team as reward (Ye, 2019).

Generally speaking, the combination of the above three paths can effectively improve the further-supporting project. As a primary factor, policy making is advantageous to adopt the "pull in" policy and promote achievement innovation and cultivation, which can attract high-level talents. The platform establishment is the key factor. A good platform can further improve the level of achievements. By the process evaluation, the purpose is to control the quality of re-research.

\subsection{Key and Difficult Issues in Research on Further-Supporting at the Later Stage of I\&E}

1) Academic science and technology competitions platforms are included in science and engineering majors, detailed mainly involving artificial science and technology, information and computer science, optoelectronic information science and engineering mechanical design manufacture and automation, industrial design, material forming and control engineering, etc. Because of that, it is an important task in an effort to overall considerate the competitions' features of each discipline and major in order to make a supporting policy that is improved, scientific, feasible, operating-friendly, and strong guidance for the further-supporting project.

2) Full consideration should be taken of the research unit's status quo of academic science and technology competitions for further improving the level of achievements of college students' academic science and technology competitions, the key point of which is to do well in "later-stage" further-supporting related research on these activities. The later-stage further-supporting is not separate from other but is the level advancing based on previous achievement so that the substantial innovation is required in operating mode. The later-stage further-supporting is to improve the quality of achievements as well as to serve as a progress for students to build interdisciplinary knowledge structure and their innovative ability.

3) As the cultivation of innovative-application-oriented talents relies on full-featured practice and training platforms and plentiful funding support, efforts should be actively taken for obtaining laboratory special funding expansion of all kinds and levels and laboratory equipment functions fitted in the competitions platforms based on full collecting the present equipment resources, which is also the key content of the research in the process of implementing the further-supporting project later.

\section{Educational and Conditions Guarantee for I\&E}

In recent years, students of science and engineering majors in Qilu University of Technology (Shandong Academy of Science) have won several prizes in so many 
competitions such as in China College Students' Entrepreneurship Competition, "Challenge Cup" National Undergraduate Curricular Academic Science and Technology Works by race, National Undergraduate Electronics Design Contest and Contemporary Undergraduate Mathematical Contest in Modeling. Thereinto, mathematics majors exclusively have harvested more than 30 state-level awards and over 200 province-level. See Table 1, the platforms for students of science and engineering. These academic science and technology platforms include mathematics, electromechanics, physics, information, etc.

Table 1. Competition platforms for encouraging I\&E for students of science and engineering (M.O.E., 2020).

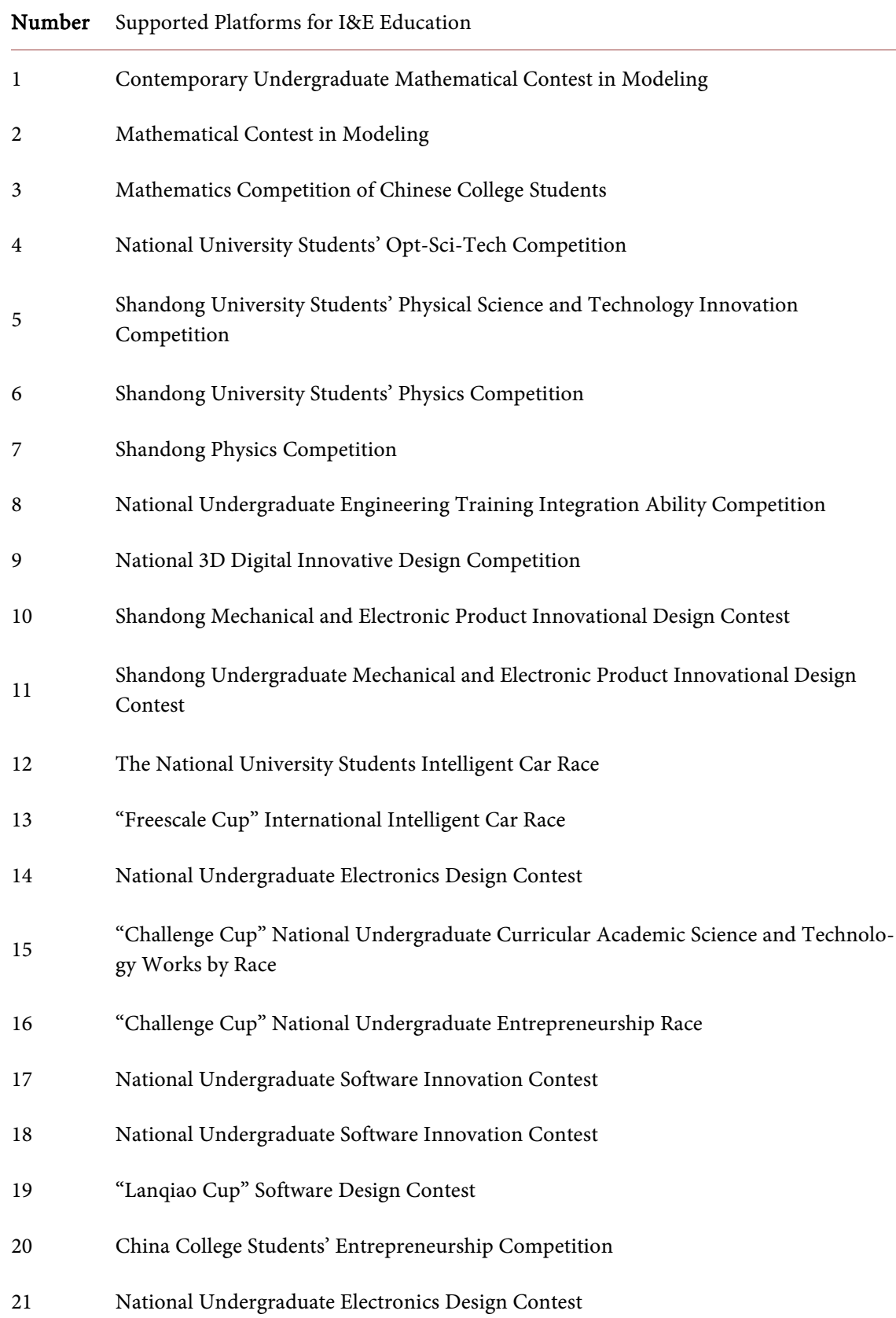


The University has devoted great efforts to the selection, organization, tutoring and instruction of these academic competitions. The related policies and incentives systems are made, which lays a sound foundation for the further-supporting the competitions.

1) Policies and Funds

A number of policies of I\&E education are made and implemented including Qilu University of Technology Interim Administration Measures for Undergraduate Curricular Competition, Qilu University of Technology Credit Management Measures for I\&E Practice, Qilu University of Technology Management Measures for Undergraduate I\&E Training Program. These policies play an important role in process of implementing academic competition competitions of science and engineering, ensuring the adequate funds for the further-supporting.

2) Systems

In order to ensure the invigoration and continuous innovation of the project construction, system of team members' admission and replacement is established. On the premise of overall team relatively stable, efforts are taken to absorb new members, especially to encourage personnel of institutes and enterprises to take part in, to support multiple disciplines crossing and the university integrated with enterprises for I\&E teaching, providing a solid guarantee for improving the team teaching and scientific research ability. Regularly hold internal experience communication meeting on team construction to deepen their understanding the significance of the team and the development of advanced technology in relevant specialties. Teachers should play their instructive role to promote the integral level of the team. Periodically invite experts to give their constructive suggestion to the team, providing the policy and technology assurance for the team construction.

Substantial and feasible incentives and restriction systems are established and improved for the team interior. The curricular group leader responsibility system is taken led by the leader of the team. Every year, part of funds is spent on supporting and rewards for members further studying and improves their academic achievements, ensuring continuous development of the team construction.

3) Conditions

In 2017, integrated with Shandong Academy of Science, Qilu University of Technology has emerged as advanced in scientific research, experiment, and practical training platforms so as to provide the self-contained practical platforms for innovation. Building the application mechanism of achievements transformation ensures the work related to academic science and technology competition competitions of science and engineering in full swing, featuring highly in cultivating students' innovative ability.

Continue to optimize and improve the running mechanism of the team and specify the orientation and guiding ideas. Depending on the scientific team of institute, taking I\&E training as the core and improving the quality of cultivation 
of application-oriented personnel as priority propel advancement of instruction level of academic science and technology competitions, bringing the task of reforming and constructing academic science and technology activities of science and engineering to a higher level.

\section{Acknowledgements}

This work is supported by the second batch of new engineering research and practice project "Construction and Practice of Regional Education Community for New Engineering Talents in Artificial Intelligence under the Background of Science and Education Integration" (project No. E-RCZN20201029 and Z2020053), Key Project of China Institute of Communication Education "Reform and Practice of New Engineering Collaborative Education Mode Based on Industry-Education Integration and School-Enterprise Cooperation" (project No. JJY 1801-51), Qilu University of Technology (Shandong Academy of Sciences) key project "Research and Practice on the Later Stage Further-Supporting and Transformation Mechanism of College Students' Academic Science and Technology Competition Achievements Based on New Engineering" (project No. 201817), Qilu University of Technology (Shandong Academy of Sciences) bidding project "Practice and Exploration of Qilu Class for Talented Training Mode under the Background of Science-Education Integration" (project No. 201803). Some researches' results of the major research topic of teaching quality reform of Shandong Jiaotong University "Research on Project Driven Innovation and Entrepreneurship Education Practice under the Background of Industry-Education Integration and New Engineering" (project No. 2019ZD02).

\section{Conflicts of Interest}

The authors declare no conflicts of interest regarding the publication of this paper.

\section{References}

Du, T. B., Yu, C. J., \& Wen, Z. (2019). Research on Optimization of College Students' Innovation and Entrepreneurship Policy Support System. Economic Review Journal, 89-95. https://doi.org/10.16528/j.cnki.22-1054/f.201909088

Fang, Z., \& Montazeritabarar, M. (2020). The Mechanism and Ethics of Academic Promotion in China's Academia. Creative Education, 11, 479-487. https://doi.org/10.4236/ce.2020.114035

Gu, J. Y. (2020). Research on the Construction of Multidimensional Innovation Practice Platform for University Students' Science and Technology Competition under the Background of "First Class Disciplines". The Science Education Article Collects, No. 3, 62-63. https://doi.org/10.16871/j.cnki.kjwhb.2020.03.028

Li, C. Y., Su, Y. Y., \& Liu, J. S. (2020). The Practice of Cultivating University Students' Professional Innovation Ability on the Platform of Competition. Science \& Technology Information, 18, 235-236. https://doi.org/10.16661/j.cnki.1672-3791.2020.04.235

Li, S. L. (2016). Research on the Policy of Promoting College Students' Innovation and 
Entrepreneurship. China University Students Career Guide, 18, 60-64.

MOE (Ministry of Education) (2020, February 21). 2019 Ranking List of National Discipline Competition in Colleges and Universities. https://www.cahe.edu.cn/site/content/11857.html

Nie, J. (2019). Research on Financing Difficulties and Countermeasures of University Students' Innovation and Entrepreneurship. Economic and Social Development, 16, 273-274.

Yan, R. S., Du, Z., Kang, K., Lv, Y., \& Sun, H. (2021). Characteristics and Practice Path of Competition-Driven Educational Mode of Scientific and Technological Innovation in Universities. Light Industry Science and Technology, 37, 187-188.

Ye, C. C. (2019). Research on University Students' Innovation and Entrepreneurship Path from the Perspective of the New Era. Knowledge Economy, 23, 148-149. https://doi.org/10.15880/j.cnki.zsji.2019.34.086

Zhang, J. (2017). “The Trinity”: Synergy Innovation Mechanism of Science and Technology-A Case Study Based on Nantong Industrial Technology Institute. Technology and Investment, 8, 44-55. https://doi.org/10.4236/ti.2017.81004 